\title{
Interleukin-22 Attenuates Acute Pancreatitis-Associated Intestinal Mucosa Injury in Mice via STAT3 Activation
}

\author{
Jinxia Bai ${ }^{1}$, Jinyun Bai ${ }^{2,3}$, and Meng Yang ${ }^{4}$ \\ ${ }^{1}$ Department of Emergency and Critical Care Medicine, Shanghai Pudong New Area People's Hospital, ${ }^{2}$ Department of Endocrinology \\ and Metabolism, Zhongshan Hospital, Fudan University, ${ }^{3}$ Fudan Institute for Metabolic Diseases, and ${ }^{4}$ Dayi Primary Education Group, \\ Shanghai, China
}

\section{Article Info}

Received July 8, 2020

Revised October 26, 2020

Accepted November 6, 2020

Published online January 28, 2021

\section{Corresponding Author}

\section{Jinxia Bai}

ORCID https://orcid.org/0000-0001-9705-0140

E-mail baijinxia1025@126.com

\begin{abstract}
Background/Aims: Interleukin-22 (IL-22) is an important cytokine maintaining homeostasis at barrier surfaces. In this study, the role of IL-22 in acute pancreatitis-associated intestinal injury was further explored.

Methods: Severe acute pancreatitis (SAP) was induced by administration of L-arginine in Balb/c mice at different time gradients. Histopathological examinations were made in both the pancreas and small intestine. Furthermore, recombinant murine IL-22 (rIL-22) was administrated to L-arginine-induced SAP mice by intraperitoneal injection. The mRNA levels of IL-22R1, Reg-III $\beta$, Reg-IIly, Bcl-2, and Bcl-xL were detected in the small intestine by real-time polymerase chain reaction, and protein levels of total and phosphorylated STAT3 were assessed via Western blot.

Results: Compared with normal control group, 72 hours of L-arginine exposure induced the most characteristic histopathological changes of SAP, evidenced by pathological changes and serum amylase levels. Meanwhile, significant pancreatitis-associated intestinal mucosa injury was also observed. The gene expression levels of antimicrobial proteins Reg-III $\beta$, Reg-IIly and anti-apoptosis proteins Bcl-2, Bcl-xL were downregulated in small intestine. Furthermore, Larginine-induced SAP was attenuated by rIL-22 treatment. Importantly, pancreatitis-associated intestinal mucosa injury was also ameliorated, reflected by improved pathological changes and significant increase in gene expression levels of Reg-III $\beta$, Reg-IIly, Bcl-2 and Bcl-xL. Consistently, serum amylase levels and mortality were decreased in mice treated with rIL-22. Mechanistically, the upregulated expressions of these protective genes were achieved by activating STAT3.

Conclusions: Exogenous rlL-22 attenuates L-arginine-induced acute pancreatitis and intestinal mucosa injury in mice, via activating STAT3 signaling pathway and enhancing the expression of antimicrobial peptides and antiapoptotic genes. (Gut Liver 2021;15:771-781)
\end{abstract}

Key Words: Interleukin-22; Pancreatitis; Intestinal mucosa; STAT3

\section{INTRODUCTION}

Acute pancreatitis is an important reason of increased morbidity and mortality worldwide, accounting for at least $30 \%$ mortality in patients who suffer from a severe pancreatitis course. ${ }^{1,2}$ Specifically, severe acute pancreatitis (SAP) often correlates to localized infection, systemic sepsis, and multiple organ dysfunction syndrome. ${ }^{3}$ Recent studies ${ }^{4,5}$ have suggested that these complications are results of intestinal mucosa barrier injuries, which increase intestinal permeability and cause bacterial translocation, subse- quently resulting in sepsis and multiple organ dysfunction syndrome.

Interleukin (IL)-22 belongs to the IL-10 cytokine family, which was first characterized in 2000 by Dumoutier et al. ${ }^{6} \mathrm{IL}-22$ is secreted by many innate and adaptive immune cells, including Th22 $\mathrm{CD} 4^{+} \mathrm{T}$ cells, Th17 $\mathrm{CD} 4^{+} \mathrm{T}$ cells, $\mathrm{CD}^{+} \mathrm{T}$ cells, dendritic cells, natural killer cells, lymphoid tissue inducer cells, and innate lymphoid cells. ${ }^{7-11}$ The receptor of IL-22 is a heterodimeric complex with two subunits, IL-22R1 and IL-10R2. Different from IL-10R2, IL-22R1 expression is confined to the surface of non-he- 
matopoietic cells. ${ }^{12}$ Therefore, innate cell populations at the sites of the gastrointestinal tract, liver, lungs and kidneys can be specifically targeted by IL-22. Mechanically, Janus kinase 1 and signal transducer and activator of transcription (STAT) 1, 3, 5 mediate IL-22 signal pathways. ${ }^{13}$ Notably, it's observed in primary cells that IL-22 phosphorylates STAT3 at the Tyr705 residue. ${ }^{12}$ Taken together, IL-22 is an immune cell-produced cytokine, regulating the function of epithelial cells and modulating the epithelial immunomicroenvironment.

Furthermore, IL-22 has the function of maintaining normal barrier homeostasis by inhibiting the systemic transmission of intestinal pathogens and symbiotic bacteria, and enhancing innate intestine epithelial defenses and mucosal barrier integrity. ${ }^{14}$ Therefore, we hypothesized that IL-22 may also protect against intestinal mucosa injury in SAP. An L-arginine-induced mice SAP model was used to test this hypothesis. Our results revealed that administration of IL-22 ameliorates L-arginine-induced SAP and associated intestinal mucosa injury, through activating the STAT3 signal and inducing downstream expression of antiapoptosis genes and antimicrobial peptides. Thus, the IL22/STAT3 pathway may become a novel therapeutic target for treating patients who suffer from acute pancreatitis and pancreatitis-associated intestinal mucosa injury.

\section{MATERIALS AND METHODS}

\section{Animal model and diets}

Eight-week-old male BALB/c (18-22 g) mice were purchased from the Experimental Animal Center of Shandong University, Jinan, China, and acclimated for a minimum of 1 week. All mice were fed with standard laboratory chow diet and watered ad libitum, with a 12-hour light/12-hour dark cycle at $23^{\circ} \mathrm{C} \pm 2{ }^{\circ} \mathrm{C}$. Mice were assigned to experimental or control group randomly. All animal experimental protocols were approved by the Shandong University Institutional Animal Care and Use Committee (IACUC number: SD1410013).

\section{Induction of experimental acute pancreatitis and treatment}

SAP was induced by two intraperitoneal injections of L-arginine hydrochloride (Sigma-Aldrich, St. Louis, MO, USA) (8\%, $\mathrm{pH}=7.0), 4 \mathrm{~g} / \mathrm{kg}$ bodyweight each, with 1-hour interval between injections as previously reported. ${ }^{15}$ Sham injections of $\mathrm{NaCl}$ were administrated to normal controls. Mice were then injected with phosphate-buffered saline (PBS) or recombinant murine IL-22 (rIL-22; $200 \mathrm{ng} /$ mouse, Miltenyi Biotec, Bergisch Gladbach, Germany) at specified times. All mice were returned to the cages, with free access to food and water. SAP group mice were sacrificed at 24, 48, and 72 hours after the second injection of L-arginine, respectively.

\section{Histological analysis}

Mice were sacrificed by cervical dislocation. Fresh specimens of pancreas or terminal small intestine were immediately fixed in $10 \%$ formalin. Tissues were embedded in paraffin, and $5 \mu \mathrm{m}$ sections were processed for hematoxylin and eosin staining. Severity of intestinal mucosal injury was evaluated based on exfoliation of epithelial cells, edema, and infiltration of inflammation cells. The mucosal thickness, crypt depth, and villous height were measured by computer software. All pathologic examinations were observed by a pathologist unaware of the treatment.

\section{RNA extraction and real-time PCR (RT-PCR)}

Total RNA was extracted from terminal small intestine using RNAiso Plus (Takara Bio Inc., Otsu, Japan) following the manufacturer's instructions. RNA was reverse-transcribed into complementary DNA using the PrimeScript ${ }^{\mathrm{TM}}$ RT Reagent Kit with gDNA Eraser (Takara Bio Inc.). RTPCR was performed using the Lightcycler480 (Roche) by the SYBR Green chemistry. The cycling conditions were as follows: $95^{\circ} \mathrm{C}$ for 2 minutes, followed by 40 cycles of $95^{\circ} \mathrm{C}$ for 10 seconds, $60^{\circ} \mathrm{C}$ for 30 seconds, and $72^{\circ} \mathrm{C}$ for 30 seconds. Messenger RNA (mRNA) expression was calculated by $2^{-\triangle \Delta \mathrm{CT}}$ method relative to housekeeping control GAPDH. ${ }^{16}$

\section{Western blot}

For Western blot analyses, terminal small intestine tissues were harvested and then homogenized in protein lysate buffer. Protein samples $(40 \mu \mathrm{g})$ were separated on precast $10 \%$ SDS polyacrylamide gels. After electrophoresis, the proteins were transferred to polyvinylidene fluoride membrane filters followed by incubation at $4^{\circ} \mathrm{C}$ overnight with primary rabbit polyclonal anti-STAT3 (1:1,000 dilution) and phosphor-STAT3 Tyr705 (1:2,000 dilution) or a mouse monoclonal anti- $\beta$-actin (1:1,000 dilution). After washing three times in Tris-buffered saline with Tween-20 (TBST), horseradish peroxidase-conjugated secondary antibodies ( $1: 5,000-1,0000$ dilution) were used in $5 \%$ to $10 \%(\mathrm{w} / \mathrm{v})$ dry non-fat milk in TBST for 1 hour at room temperature. Finally, membranes were washed with TBST and visualized with a chemiluminescence reagent (ECL Millipore Biotechnology Inc., Burlington MA, USA) and quantified using ImageJ Analysis Software. 


\section{Statistical analysis}

Nonparametric tests including the Mann-Whitney U tests between two group or one-way analysis of variance among three and more group were used for statistical analyses. p-value $<0.05$ was considered significant. Data were analyzed with GraphPad Prism software, version 8.0 (GraphPad Software Inc., La Jolla, CA, USA).

\section{RESULTS}

\section{L-arginine-induced characteristic severe acute pancreatitis}

Mice were injected with L-arginine or $\mathrm{NaCl}$ control with two intraperitoneal injections ( $4 \mathrm{~g} / \mathrm{kg}$ ), 1 hour apart. Mice injected with L-arginine developed SAP and were divided into three groups by time gradients: SAP 24-hour group, SAP 48-hour group, and SAP 72-hour group (Fig. 1A). Significant pancreatic histopathological changes were observed in the SAP group. In detail, 24 hours after L- arginine administration, pancreatic interstitial edema was seen, with a small number of inflammatory cells infiltrated, and partial pancreatic lobes destructed. Necrosis mostly occurred at edge of pancreatic lobules (SAP 24 hours) and gradually extended to the center (SAP 48 hours). By 72 hours, the color of pancreas turned into gray, only a few normal pancreatic lobules remained and large necrosis areas appeared, with a large number of inflammatory cells infiltrated (Fig. 1B and C). Consistently, Fig. 1D shows the changes of serum amylase activity in each group of mice, with a trend of initial increase and then decrease over time in the SAP group. At the most severe time point of histopathological change, that is, SAP 72-hour group, the serum amylase decreased, which was consistent with the decline of the serum amylase in patients with acute severe pancreatitis. Taken together, these results suggest that L-arginine injection induces significant mice SAP model, and 72 hours after L-arginine injection is the optimal timepoint for SAP induction.
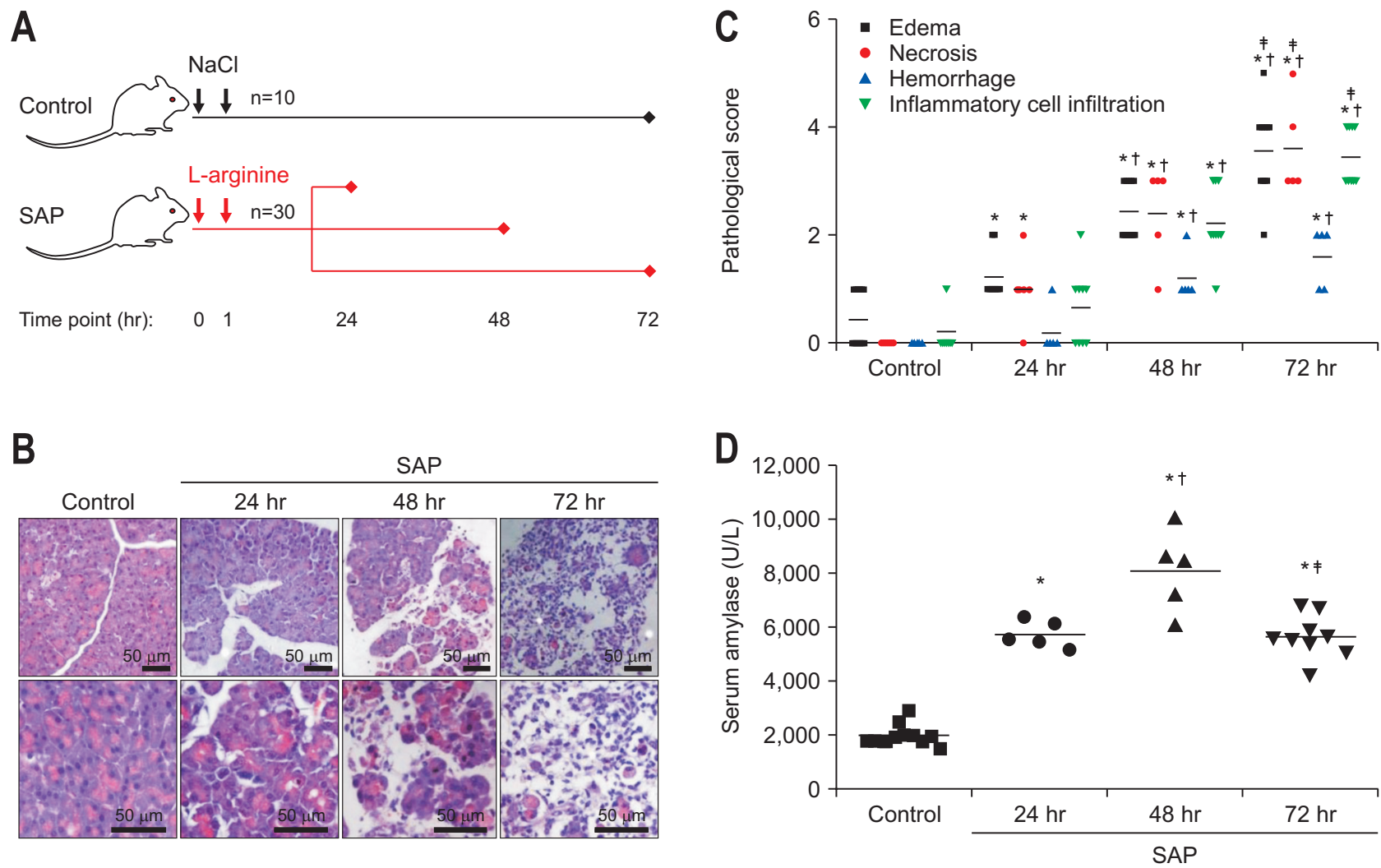

Fig. 1. Representative pathological/morphological changes in pancreatic tissue and serum amylase activity in different groups. (A) Mice received two intraperitoneal injections of L-arginine or $\mathrm{NaCl}$ solution $(4 \mathrm{~g} / \mathrm{kg}) 1$ hour apart. (B) Representative pathological morphology changes in different groups: normal control group, SAP 24-hour group, SAP 48-hour group, and SAP 72-hour group (H\&E). (C) Pathological changes in edema, necrosis, hemorrhage, and inflammatory cell infiltration were evaluated. (D) Serum amylase activity in different groups.

SAP, severe acute pancreatitis. ${ }^{*} p<0.05$ versus normal control; ${ }^{\dagger} p<0.05$ versus SAP 24 -hour group; ${ }^{\ddagger} p<0.05$ versus SAP 48 -hour group (n=7-10). Data are presented as individual scatter dots with the mean values. 


\section{Acute pancreatitis-associated intestinal mucosa injury was observed in L-arginine-induced SAP}

Histopathological observation of the hematoxylin and eosin samples of terminal small intestine tissues revealed significant epithelial cells exfoliation, villi edema and broadening, and inflammatory cell infiltration in SAP group (Fig. 2B-D). By contrary, above histological changes were absent in the normal control group (administration of $\mathrm{NaCl}$ alone) (Fig. 2A). Importantly, the severity of pathological changes was positively correlated with the time gradients of L-arginine administration. Furthermore, the mucosal thickness, villus height, and crypt depth in SAP group were significantly lower than normal control, and these pathological changes were most severe in SAP 72hour group (Fig. 2E). These results reveal that L-arginine injection also induces acute pancreatitis-associated intestinal mucosa injury.

\section{3. rlL-22 treatment improved acute pancreatitis and pancreatitis-associated intestinal mucosa injury}

Mice SAP models were successfully induced by L-arginine injection, and the mice were sacrificed 72 hours after the L-arginine administration. Notably, from 48 hours before L-arginine injection to sacrifice, mice received several intraperitoneal injections of PBS or rIL-22 at the indicated times (Fig. 3A). Representative hematoxylin and eosin sections of intestinal tissues were shown and pathological changes including mucosal thickness, crypt depth and villous height of intestinal mucosa were analyzed. As a result, treatment with rIL-22 could reduce the severity of intesti- nal mucosal injury (Fig. 3B) and improve these pathohistological parameters (Fig. 3C). Moreover, compared PBS treatment, histopathological improvements in the pancreas were observed in the rIL-22 treatment mice (Fig. 3D), and the serum amylase of the rIL-22-treated mice decreased significantly (Fig. 3E). In addition, the mortality of rIL22 treated mice was significantly lower than of the PBStreated mice (Fig. 3F). Together, rIL-22 treatment shows good performance in improvement of acute pancreatitis and acute pancreatitis-associated intestinal mucosa injury.

\section{4. rIL-22 treatment upregulated antimicrobial and antiapoptosis genes' expression levels}

It is known that in gut mucosal injury models, inflammatory processes were regulated by the antimicrobial proteins (AMPs) Reg-III $\beta$ and Reg-III $\gamma$. To determine whether L-arginine-induced SAP affects expression of Reg-III $\beta$ and Reg-III $\gamma$, their mRNA levels in terminal small intestine tissues of L-arginine-induced SAP were detected by RT-PCR. As demonstrated in Fig. 4A, compared with the normal control group, Reg-III $\beta$ and Reg-III $\gamma$ mRNA levels were markedly decreased, especially in the 72-hour group. Consistently, mRNA levels of antiapoptosis proteins Bcl-2 and $\mathrm{Bcl}-\mathrm{xL}$ were also significantly downregulated in the SAP group (Fig. 4B). Moreover, as shown in Fig. 4C, mRNA levels of IL-22R1 increased significantly in 24 and 48 hours L-arginine-induced SAP models, which were in agreement with the acute pancreatitis model induced by intraperitoneal injection of caerulein and feeding with cholinedeficient diet supplemented with DL-ethionine. ${ }^{17}$
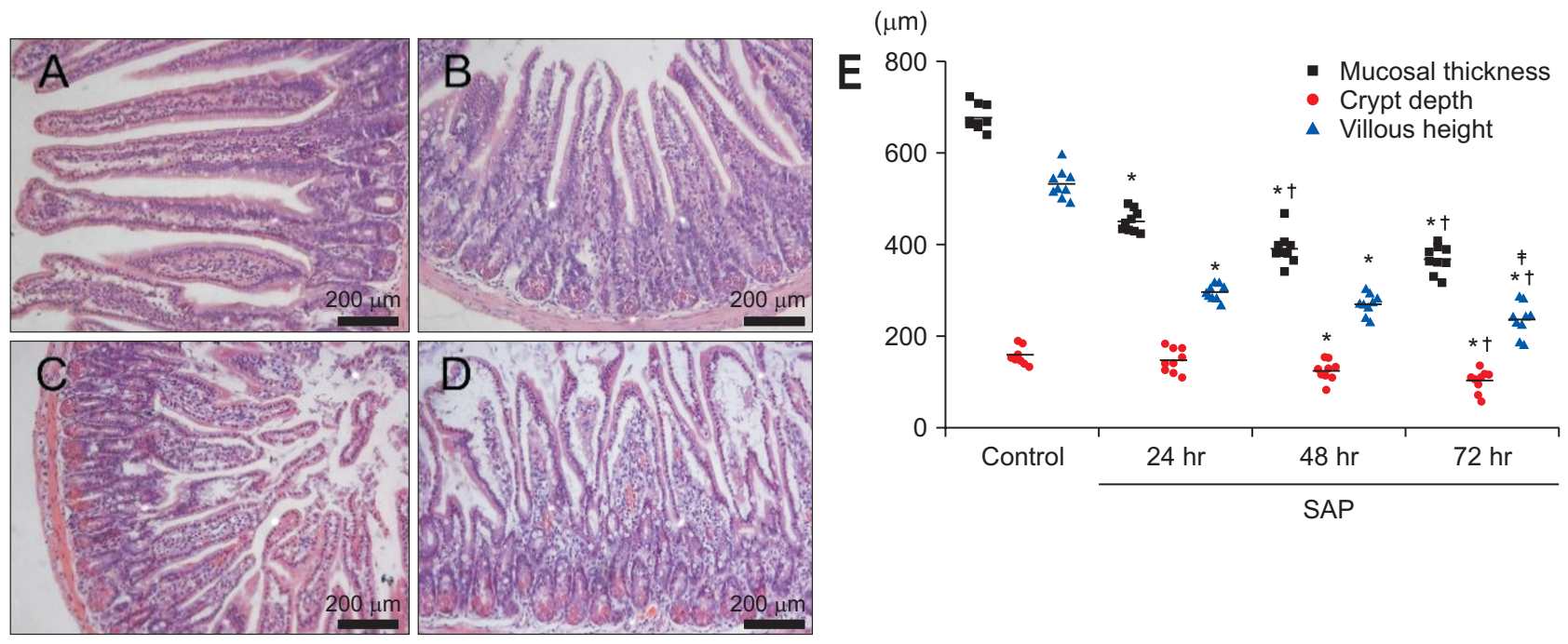

Fig. 2. Histological changes and pathohistological parameters in the small intestine. (A-D) Representative H\&E staining of intestinal tissue sections from the normal control group (A) and the SAP 24-hour (B), 48-hour (C), and 72-hour groups (D) after the second injection of L-arginine is shown. (E) Changes in mucosal thickness, crypt depth and villous height of the intestinal mucosa are shown.

SAP, severe acute pancreatitis. ${ }^{*} p<0.05$ versus normal control group; ${ }^{\dagger} p<0.05$ versus SAP 24 -hour group; ${ }^{\ddagger} p<0.05$ versus SAP 48 -hour group ( $n=7-$ 10). Data are presented as individual scatter dots with the mean values. 

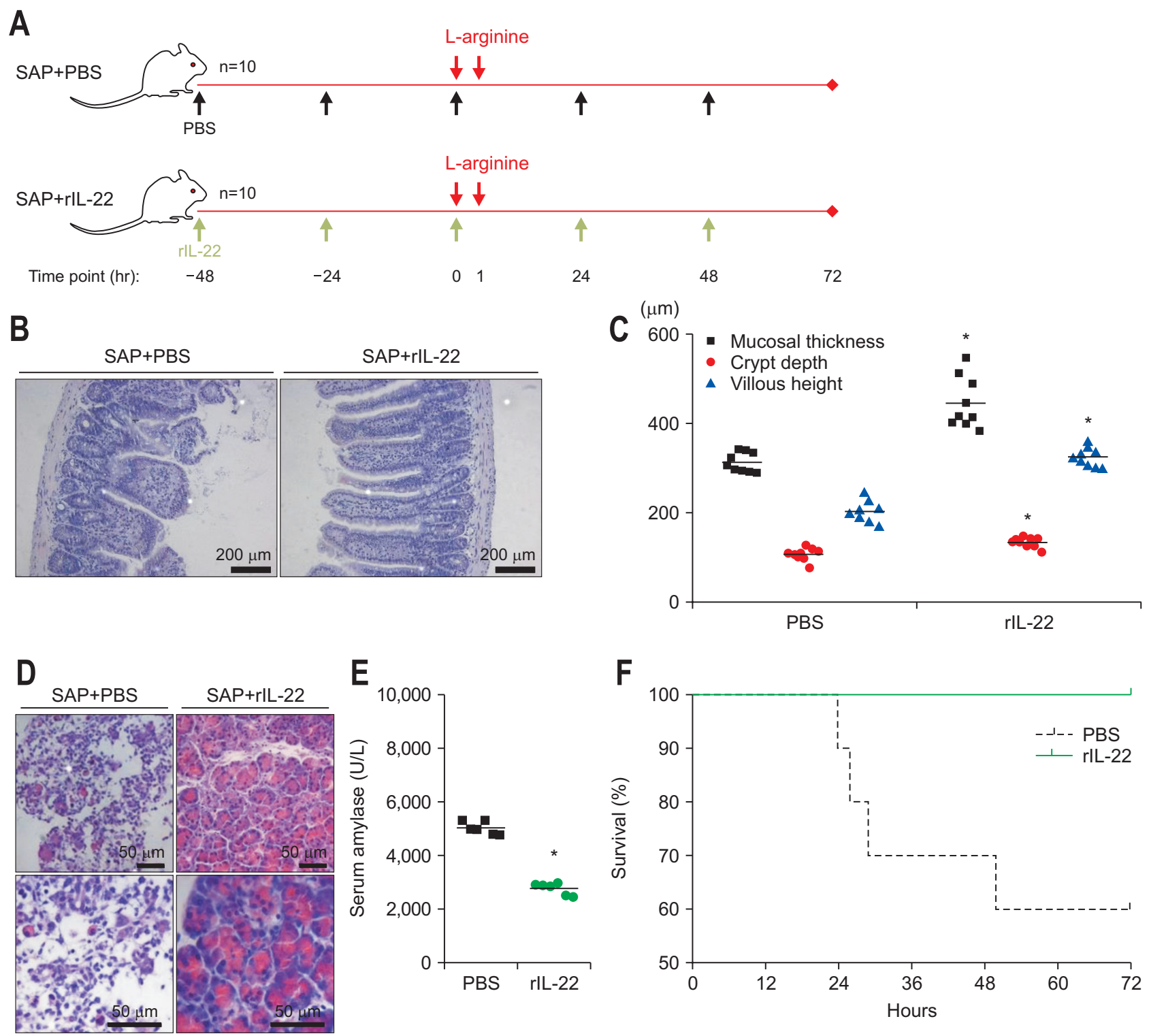

Fig. 3. Treatment with rlL-22 protein ameliorates acute pancreatitis and acute pancreatitis-associated intestine mucosa injury in L-arginineinduced SAP. (A) BALB/c mice underwent L-arginine-induced SAP. PBS or rIL-22 was administered to mice at the indicated times (arrows). (B) Representative H\&E staining of intestinal tissue is shown. (C) Mucosal thickness, crypt depth and villous height of the intestinal mucosa are significantly higher in the rlL-22 treatment group than in the PBS treatment group. (D) Representative H\&E staining of pancreatic tissue from the rlL-22 or PBS treatment group is shown. (E) Serum amylase levels in SAP mice after PBS or rIL-22 treatment. (F) Percent survival in the rIL-22 or PBS treatment group is shown.

rIL-22, recombinant murine interleukin-22; SAP, severe acute pancreatitis; PBS, phosphate-buffered saline. ${ }^{*} p<0.05$ versus the PBS treatment group ( $\mathrm{n}=7-10)$. Data are presented as individual scatter dots with the mean values.

Recent studies indicated that IL-22 was an important regulatory cytokine in maintaining gut epithelial integrity and mucosal immunity. ${ }^{18}$ Therefore, in this study, the effect of exogenous IL-22 supplementation on modulating those genes expression in small intestine during $\mathrm{L}$-arginineinduced SAP was explored. Consistently, rIL-22 administration significantly enhanced the mRNA expression of Reg-III $\beta$ and Reg-III $\gamma$ genes which were known to play important role in tissue regeneration (Fig. 4D). Moreover, rIL-22 administration also increased the mRNA expres- sion of $\mathrm{Bcl}-2$ and $\mathrm{Bcl}-\mathrm{xL}$ in intestine tissues of $\mathrm{L}$-arginineinduced SAP mice (Fig. 4E). Bcl-2 and Bcl-xL proteins are members of $\mathrm{Bcl}-2$ protein family, which is known play regulatory role in inhibiting cell apoptosis. ${ }^{19}$ Besides, IL-22R1 expression was slightly increased following systemic rIL22 administration (Fig. 4F). Together, these results indicate that rIL-22 supplement upregulates the gene expression levels of antimicrobial and antiapoptosis proteins in mice intestines. 

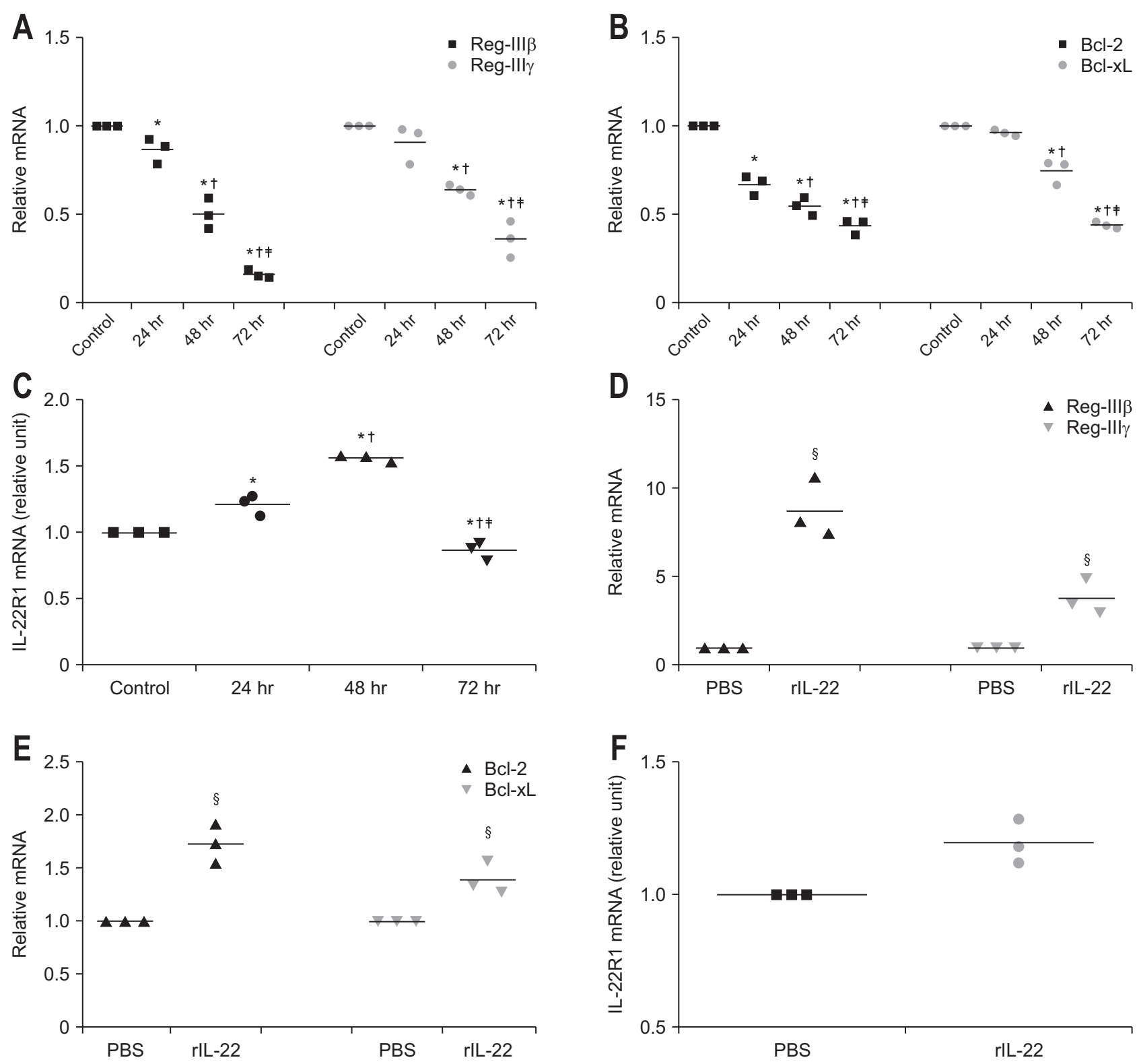

Fig. 4. Decreased expression levels of antiapoptosis genes in intestinal tissues of SAP mice are upregulated by rIL-22 administration. A mouse model of SAP was induced via the administration of L-arginine. After 24,48 , and 72 hours of two L-arginine administrations, small intestine RNA was extracted, and cDNA was prepared for real-time PCR analysis. The results are shown as fold changes in Reg-IIIB and Reg-IIly (A); Bcl-2 and $\mathrm{Bcl}-\mathrm{xL}(\mathrm{B})$ and IL-22R1 (C) mRNA expression relative to that of the control group. Subsequently, the 72 hour-induced-SAP model was chosen for further investigation. Mice were given PBS or rlL-22 (200 ng/mouse $\times 5$ times) (Fig. $3 A$ ) intraperitoneally. After 72 hours of L-arginine administration, intestine RNA was isolated, and cDNA was prepared for real-time PCR analysis. The results are shown as fold changes in Reg-IIIB and RegIIly (D); Bcl-2 and Bcl-xL (E) and IL-22R1 (F) mRNA expression relative to that of the control group.

SAP, severe acute pancreatitis; rIL-22, recombinant murine interleukin-22; cDNA, complementary DNA; PCR, polymerase chain reaction; mRNA, messenger RNA; PBS, phosphate-buffered saline. ${ }^{*} p<0.05$ versus the normal control group; ${ }^{\dagger} p<0.05$ versus the SAP 24 -hour group; ${ }^{\ddagger} p<0.05$ versus the SAP 48-hour group. ${ }^{\S} \mathrm{p}<0.05$ versus the PBS treatment group ( $\left.\mathrm{n}=7-10\right)$. Data are presented as individual scatter dots with the mean values.

\section{5. rIL-22 induces phosphorylation of STAT3}

It has been shown that IL-22 activated the STAT3 signaling pathway in colonic epithelial cells, pancreatic acinar cells, and hepatocytes. ${ }^{20,21}$ To examine whether rIL-22 modulates intestinal mucosa function through activating the STAT3 signaling pathway, terminal intestine tissue was harvested 72 hours after L-arginine injection. By means of
Western blot analysis, phosphorylated STAT3 was detected using a phosphor-STAT3 Tyr705 specific antibody. Although levels of total STAT3 proteins did not change, rIL22 treatment significantly increased protein levels of phosphorylated STAT3 (Fig. 5A). Besides, the phosphor-STAT3 Tyr705/total STAT3 ratio was markedly much higher in rIL-22 treatment group compared with PBS treatment 

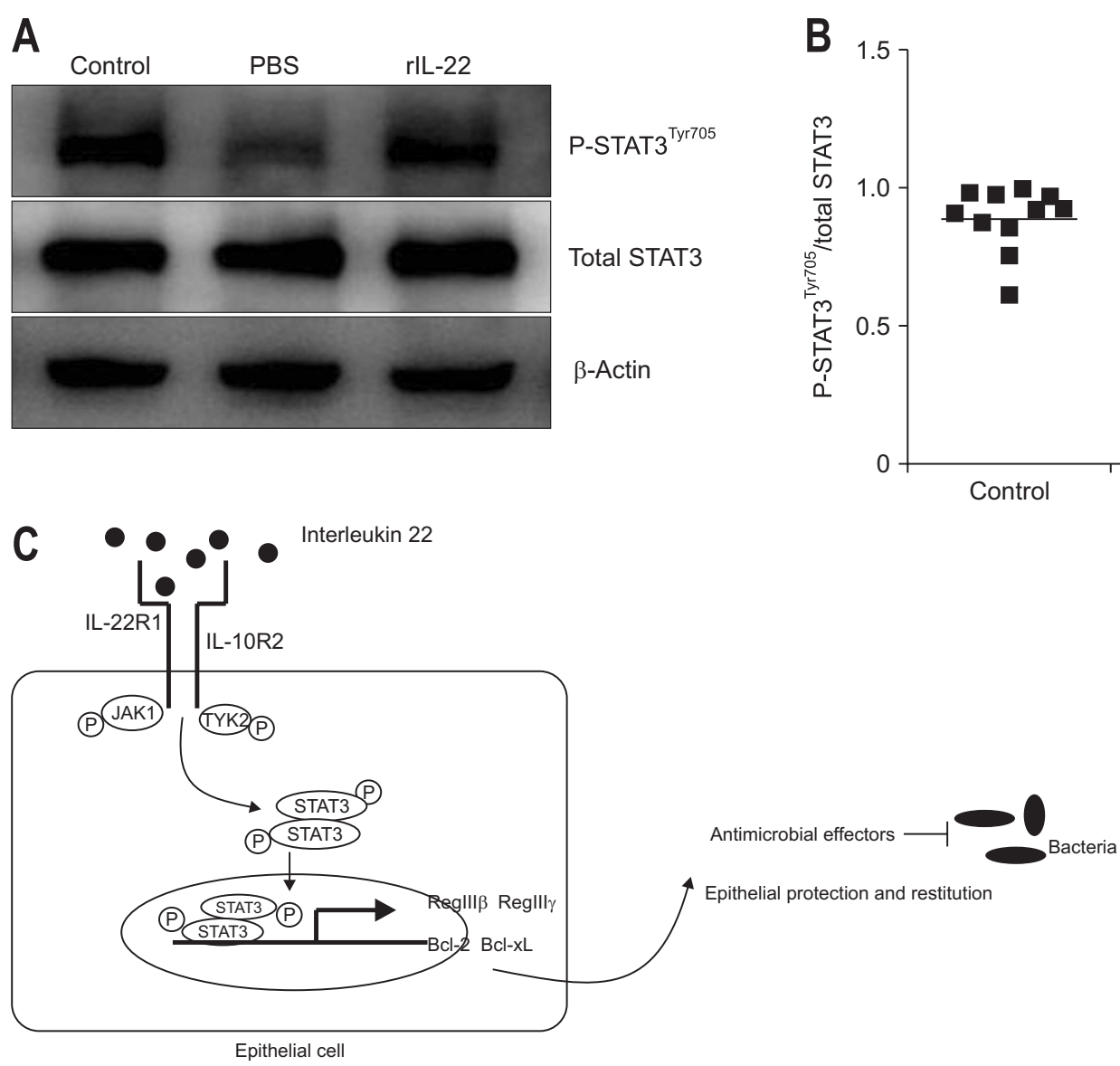

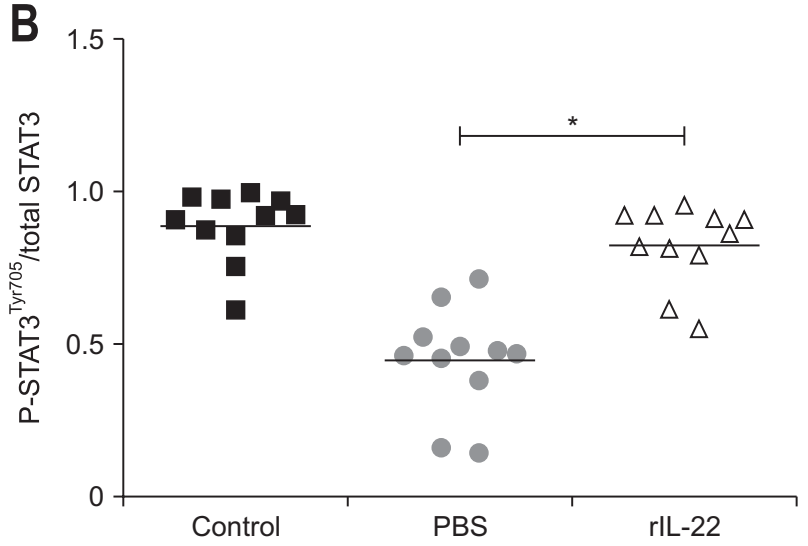

Fig. 5. rIL-22 induces STAT3 phosphorylation in the small intestine and the proposed model. (A) STAT3 expression and activation (phosphorylation of Tyr705) were examined by Western blotting using total tissue lysates from the small intestines of rlL-22- and PBS-treated mice 72 hours after the injection of L-arginine. (B) The relative ratios of phosphor-STAT3 to total STAT3 are shown. There is a significant increase in phosphor-STAT3 levels in the rIL-22 treatment group. Data are presented as individual scatter dots with the mean values, $\mathrm{n}=3$ in each group. (C) Proposed model for which the IL-22/STAT3 pathway attenuates acute pancreatitis-associated intestinal mucosa injury. Exogenous recombinant murine IL-22 stimulates the IL-22R1/IL-10R2 receptor complex, activating the STAT3 signaling pathway in intestinal epithelial cells. Activated STAT3 stimulates epithelial cells to produce the antimicrobial proteins Reg-IIIB and Reg-IIly and the antiapoptosis proteins Bcl-2 and Bcl-xL, which subsequently results in tissue repair and homeostasis maintenance in the intestine.

rIL-22, recombinant murine interleukin-22; STAT3, signal transducer and activator of transcription 3; PBS, phosphate-buffered saline; JAK, Janus kinase. ${ }^{*} p<0.05$.

group (Fig. 5B). Hence, we propose the regulatory role of IL-22/STAT3 pathway in attenuating acute pancreatitisassociated intestinal mucosa injury (Fig. 5C). Exogenous rIL-22 stimulates IL-22R1/IL-10R2 receptor complex, activating STAT3 signaling pathway in intestinal epithelial cells (IECs), and hereafter stimulates epithelial cells to produce AMPs Reg-III $\beta$, Reg-III $\gamma$ and antiapoptosis proteins $\mathrm{Bcl}-2, \mathrm{Bcl}-\mathrm{xL}$, which subsequently resulting in tissue repair and homeostasis maintenance in the intestine.

\section{DISCUSSION}

There is overwhelming evidence that SAP is combined with different degree of multiple organ dysfunction syndrome. ${ }^{22,23}$ Moreover, some clinical and experimental stud- ies $^{24,25}$ have shown that intestinal mucosa injury is a critical part in the progression of SAP. Consistently, in this study, we find that the villous height, crypt depth and mucosal thickness of small intestine tissue in SAP group gradually decrease along with the disease progression.

Exclusively produced by leukocytes, IL-22 is proposed as a specialized cytokine messenger mediating the crosstalk between leukocytes and epithelial cells. A heterodimer complex consisting of IL-22R1 and IL-10R2 is IL-22 receptor. ${ }^{13}$ Among the two elements, IL-10R2 is ubiquitously expressed in various organs, whereas IL-22R1 expression is limited to epithelial cells with high levels of messenger RNA reported in the pancreas and small intestines. ${ }^{12,26}$ This expression pattern of IL-22R1 suggested that the gastrointestinal system should be highly responsive to IL-22. Subsequently, the expression levels of IL-22R1 in the small 
intestine before and after SAP induction were detected. Consistent with our hypothesis, levels of IL-22R1 expression are upregulated early in the ongoing SAP, and then decrease, indicating that IL-22R1 expression is a stress inducible pattern. Since IL-22R1 is highly expressed in IECs, decreased IL-22R1 suggests that IECs were shedding with the persistent damage of intestine mucosa in SAP.

Recent studies have shown that IL-22 has regulatory function in epithelial cells, including stimulating epithelial cells to produce a wide variety of AMPs, reinforcing the mucus barrier by inducing mucin production, enhancing the epithelial regeneration with goblet cell restitution, and promoting intestinal wound healing. ${ }^{27,28}$ The key AMPs family of the small intestine is the regenerating gene (Reg) family of C-type lectins, ${ }^{29}$ including Reg-III $\beta$ and Reg-III $\gamma$, which are recognized for their role in intestinal mucosa barrier integrity. ${ }^{27,30}$ IL-22 knockout mice are highly susceptible to Citrobacter rodentium infection, along with increased intestinal epithelial damage, systemic bacterial burden and mortality. ${ }^{27}$ Exogenous mouse Reg-III $\gamma$ can increase survival of IL-22 knockout mice after infection. Besides, IL-22 induces secretion of AMPs from IECs, including Reg-III $\beta$ and Reg-III $\gamma$. Furthermore, Rendon et al. ${ }^{31}$ found that the treatment with IL-22 attenuated the increase of intestinal permeability and reduced gut bacterial load after alcohol exposure. Consistent with these studies, our data indicated that rIL-22 treatment upregulates AMPs expression and protects gut epithelial barrier in SAP-associated intestinal mucosa injury. Mechanically, Reg-III $\gamma$ can kill Gram-positive bacteria through recognizing peptidoglycan, damaging the cell wall and provoking cytoplasmic leakage. ${ }^{30,32}$ Reg-III $\gamma$ is also necessary for providing the spatial segregation to separate the microbiota from host small intestinal epithelial surface. ${ }^{33}$ Besides, though the killing Gram-negative bacteria mechanism of Reg-III $\beta$ is not fully understood, a recent study found that permeabilizing outer membrane is an initial step, followed by triggering bacterial death. ${ }^{34}$ In addition, Sugimoto et al. ${ }^{35}$ demonstrated that IL-22 enhances the production of membrane-bound mucins which are involved in mucus layer formation to prevent invasion by luminal bacteria and contributes to facilitate goblet cell restitution in intestinal inflammation. Taken together, these data highlight that IL-22 is a critical regulatory cytokine which helps maintaining normal barrier homeostasis via enhancement of antimicrobial response and tissue repair ability at intestine mucosa surfaces.

In addition to antimicrobial and tissue repair mechanisms in small intestine tissue, IL-22 mediates further protection by activating antiapoptotic pathway. Apoptosis is defined as "programmed cell death," which has important role in the intestinal homeostasis. However, excessive apoptosis is harmful and may lead to tissue injuries in pathological conditions including radiation, ${ }^{36}$ ischemia and ischemia/reperfusion ${ }^{37}$-induced intestinal injury. Several studies ${ }^{4,38,39}$ have demonstrated that apoptosis was accelerated in IECs and inhibition of apoptosis could prevent intestinal epithelial injury. Our study found that Bcl-2 and $\mathrm{Bcl}-\mathrm{xL}$ levels in small intestinal tissues are decreased in the $\mathrm{L}$-arginine-induced SAP. $\mathrm{Bcl}-2$ and $\mathrm{Bcl}-\mathrm{xL}$ proteins are the important antiapoptotic proteins in the $\mathrm{Bcl}-2$ protein family. ${ }^{40}$ Therefore, our experiment indirectly suggests that apoptosis in intestinal epithelium is increased in the SAP mice model. In 2004, Pan et al. ${ }^{41}$ first demonstrated that IL22 is a critical survival factor for hepatocytes via upregulating antiapoptotic (e.g., Bcl-2, Bcl-xL, Mcl-1) and mitogenic (e.g., c-myc, cyclin D1, Rb2, CDK4) proteins. Lately, Feng et al. ${ }^{42}$ suggested that IL-22 pretreatment attenuated the severity of caerulein-induced acute pancreatitis through directly increasing the expression of $\mathrm{Bcl}-2$ and $\mathrm{Bcl}-\mathrm{xL}$, which bind to beclin1 protein to inhibit autophagosome formation and also exert antiapoptotic effect. Furthermore, Xue et al. ${ }^{18}$ also reported that isolated primary pancreatic acinar cells showed delayed spontaneous apoptosis when treated with IL-22 in vitro.

Therefore, we hypothesize that supplement of IL-22 protects the intestinal barrier integrity from downregulating the excessive apoptosis. Consistently, we showed that rIL-22-treated SAP mice have high levels of Bcl-2 and Bcl$\mathrm{xL}$ with alleviated intestinal mucosa damage. Besides, IL22 showed the role of tissue repairing in mucosal wound healing. ${ }^{28}$ In our study, rIL-22 treatment increased mRNA expression of IL-22R1 gene in small intestine tissue, compared with the PBS treatment. We suggest that reconstitution of IL-22R1 by IL-22 treatment is due to the recovery of IECs. Our next aim was to identify the signal pathway IL-22 mediated to induce the expression of antimicrobial and antiapoptosis genes.

The IL-22/STAT3 signaling axis has been revealed in human colonic biopsies, mouse pancreas tissues, and primary mouse pancreatic acinar cells and hepatocytes. ${ }^{18,28,43-46}$ It depends on IL-22-specific receptor IL-22R1, which is restricted to nonhematopoietic cells of intestine, pancreas, liver, lung and kidney. ${ }^{12}$ Concretely, previous studies have found that mice with IECs-specific STAT3 knockout showed greatly downregulated expression of Reg-III $\beta$ and Reg-III $\gamma$, and reduced antiapoptosis gene expression. Most importantly, IL-22 knockout mice showed an almost complete lack of epithelial STAT3 activity. ${ }^{28}$ Above data indicate that in IECs, IL-22/STAT3 pathway regulates mucosal wound healing. Moreover, IL-22 induced the expression of $\mathrm{Bcl}-2, \mathrm{Bcl}-\mathrm{xL}$ in a STAT3-dependent manner to ameliorate caerulein-induced pancreatitis ${ }^{42}$ and protect hepatocytes 
from alcoholic liver injury. ${ }^{47}$ Our Western blot data reveals that in small intestine tissues, IL-22 treatment group has significantly higher expression level of phosphorylated STAT3 than PBS treatment group. Thus, we concluded that upregulated expressions of Reg-III $\beta$, Reg-III $\gamma$ and Bcl-2, Bcl-xL after rIL-22 treatment may due to activated STAT3 signaling pathway induced by IL-22.

In conclusion, as depicted and summarized in Fig. 5C, our study highlights that application of exogenous rIL22 attenuates intestinal mucosa injury by upregulating the expression of Reg-III $\beta$, Reg-III $\gamma$ and Bcl-2, Bcl-xL through activation of the STAT3 signal in L-arginine-induced SAP mice model. Therefore, IL-22 supplement may serve as a potential therapeutic strategy for patients who suffer from SAP.

\section{CONFLICTS OF INTEREST}

No potential conflict of interest relevant to this article was reported.

\section{ACKNOWLEDGEMENTS}

This work has been supported by the Shandong Provincial Science and Technology Committee of China (2014GGH218034). We are grateful to Hongwei Xu (Shandong Provincial Hospital Affiliated to Shandong University, Jinan, China) for experimental design guidance.

\section{AUTHOR CONTRIBUTIONS}

Experiment design: Jinxia B. Acquisition and analysis of data: Jinyun B., M.Y. Reagents and material support: Jinxia B. Writing - original draft: Jinxia B. Approval of final manuscript: all authors.

\section{ORCID}

Jinxia Bai https://orcid.org/0000-0001-9705-0140 Jinyun Bai https://orcid.org/0000-0002-1287-8457 Meng Yang https://orcid.org/0000-0003-0063-7100

\section{REFERENCES}

1. Gravante G, Garcea G, Ong SL, et al. Prediction of mortality in acute pancreatitis: a systematic review of the published evidence. Pancreatology 2009;9:601-614.

2. Harper SJ, Cheslyn-Curtis S. Acute pancreatitis. Ann Clin Biochem 2011;48(Pt 1):23-37.

3. Petrov MS, Shanbhag S, Chakraborty M, Phillips AR, Windsor JA. Organ failure and infection of pancreatic necrosis as determinants of mortality in patients with acute pancreatitis. Gastroenterology 2010;139:813-820.

4. Yasuda T, Takeyama Y, Ueda T, et al. Breakdown of intestinal mucosa via accelerated apoptosis increases intestinal permeability in experimental severe acute pancreatitis. J Surg Res 2006;135:18-26.

5. Sun X, Shao Y, Jin Y, et al. Melatonin reduces bacterial translocation by preventing damage to the intestinal mucosa in an experimental severe acute pancreatitis rat model. Exp Ther Med 2013;6:1343-1349.

6. Dumoutier L, Lejeune D, Colau D, Renauld JC. Cloning and characterization of IL-22 binding protein, a natural antagonist of IL-10-related T cell-derived inducible factor/IL-22. J Immunol 2001;166:7090-7095.

7. Seiderer J, Brand S. IL-22: a two-headed cytokine in IBD? Inflamm Bowel Dis 2009;15:473-474.

8. Witte E, Witte K, Warszawska K, Sabat R, Wolk K. Interleukin-22: a cytokine produced by T, NK and NKT cell subsets, with importance in the innate immune defense and tissue protection. Cytokine Growth Factor Rev 2010;21:365-379.

9. Ouyang W, Rutz S, Crellin NK, Valdez PA, Hymowitz SG. Regulation and functions of the IL-10 family of cytokines in inflammation and disease. Annu Rev Immunol 2011;29:71109.

10. Sonnenberg GF, Fouser LA, Artis D. Border patrol: regulation of immunity, inflammation and tissue homeostasis at barrier surfaces by IL-22. Nat Immunol 2011;12:383-390.

11. Zenewicz LA, Flavell RA. Recent advances in IL-22 biology. Int Immunol 2011;23:159-163.

12. Wolk K, Kunz S, Witte E, Friedrich M, Asadullah K, Sabat R. IL-22 increases the innate immunity of tissues. Immunity 2004;21:241-254.

13. Xie MH, Aggarwal S, Ho WH, et al. Interleukin (IL)-22, a novel human cytokine that signals through the interferon receptor-related proteins CRF2-4 and IL-22R. J Biol Chem 2000;275:31335-31339.

14. Sonnenberg GF, Monticelli LA, Alenghat T, et al. Innate lymphoid cells promote anatomical containment of lymphoidresident commensal bacteria. Science 2012;336:1321-1325.

15. Dawra R, Sharif R, Phillips P, Dudeja V, Dhaulakhandi D, Saluja AK. Development of a new mouse model of acute pancreatitis induced by administration of L-arginine. Am J Physiol Gastrointest Liver Physiol 2007;292:G1009-G1018.

16. Livak KJ, Schmittgen TD. Analysis of relative gene expression data using real-time quantitative PCR and the 2(-Delta Delta C(T)) Method. Methods 2001;25:402-408. 
17. Velusamy RK, Tamizhselvi R. Protective effect of methylsulfonylmethane in caerulein-induced acute pancreatitis and associated lung injury in mice. J Pharm Pharmacol 2018;70:1188-1199.

18. Xue J, Nguyen DT, Habtezion A. Aryl hydrocarbon receptor regulates pancreatic IL-22 production and protects mice from acute pancreatitis. Gastroenterology 2012;143:16701680 .

19. Ashkenazi A, Fairbrother WJ, Leverson JD, Souers AJ. From basic apoptosis discoveries to advanced selective BCL-2 family inhibitors. Nat Rev Drug Discov 2017;16:273-284.

20. Glal D, Sudhakar JN, Lu HH, et al. ATF3 sustains IL22-induced STAT3 phosphorylation to maintain mucosal immunity through inhibiting phosphatases. Front Immunol 2018;9:2522.

21. Radaeva S, Sun R, Pan HN, Hong F, Gao B. Interleukin 22 (IL-22) plays a protective role in $\mathrm{T}$ cell-mediated murine hepatitis: IL-22 is a survival factor for hepatocytes via STAT3 activation. Hepatology 2004;39:1332-1342.

22. Mounzer R, Langmead CJ, Wu BU, et al. Comparison of existing clinical scoring systems to predict persistent organ failure in patients with acute pancreatitis. Gastroenterology 2012;142:1476-1482.

23. Mole DJ, Webster SP, Uings I, et al. Kynurenine-3-monooxygenase inhibition prevents multiple organ failure in rodent models of acute pancreatitis. Nat Med 2016;22:202-209.

24. Lu Y, Yu Y, Yang M, Liu H, Li B, Xu H. Protective effects of saizen in combination with stilamin on intestinal mucosa of a rabbit model of severe acute pancreatitis. Pancreas 2013;42:102-107.

25. Juvonen PO, Alhava EM, Takala JA. Gut permeability in patients with acute pancreatitis. Scand J Gastroenterol 2000;35:1314-1318.

26. Gurney AL. IL-22, a Th1 cytokine that targets the pancreas and select other peripheral tissues. Int Immunopharmacol 2004;4:669-677.

27. Zheng Y, Valdez PA, Danilenko DM, et al. Interleukin-22 mediates early host defense against attaching and effacing bacterial pathogens. Nat Med 2008;14:282-289.

28. Pickert G, Neufert C, Leppkes M, et al. STAT3 links IL-22 signaling in intestinal epithelial cells to mucosal wound healing. J Exp Med 2009;206:1465-1472.

29. Gallo RL, Hooper LV. Epithelial antimicrobial defence of the skin and intestine. Nat Rev Immunol 2012;12:503-516.

30. Cash HL, Whitham CV, Behrendt CL, Hooper LV. Symbiotic bacteria direct expression of an intestinal bactericidal lectin. Science 2006;313:1126-1130.

31. Rendon JL, Li X, Akhtar S, Choudhry MA. Interleukin-22 modulates gut epithelial and immune barrier functions following acute alcohol exposure and burn injury. Shock 2013;39:11-18
32. Lehotzky RE, Partch CL, Mukherjee S, et al. Molecular basis for peptidoglycan recognition by a bactericidal lectin. Proc Natl Acad Sci U S A 2010;107:7722-7727.

33. Vaishnava S, Yamamoto M, Severson KM, et al. The antibacterial lectin RegIIIgamma promotes the spatial segregation of microbiota and host in the intestine. Science 2011;334:255258.

34. Miki T, Hardt WD. Outer membrane permeabilization is an essential step in the killing of Gram-negative bacteria by the lectin RegIII $\beta$. PLoS One 2013;8:e69901.

35. Sugimoto K, Ogawa A, Mizoguchi E, et al. IL-22 ameliorates intestinal inflammation in a mouse model of ulcerative colitis. J Clin Invest 2008;118:534-544.

36. Mylonas PG, Matsouka PT, Papandoniou EV, Vagianos C, Kalfarentzos F, Alexandrides TK. Growth hormone and insulin-like growth factor I protect intestinal cells from radiation induced apoptosis. Mol Cell Endocrinol 2000;160:115122.

37. Ikeda H, Suzuki Y, Suzuki M, et al. Apoptosis is a major mode of cell death caused by ischaemia and ischaemia/ reperfusion injury to the rat intestinal epithelium. Gut 1998;42:530-537.

38. Wang X, Wang B, Wu J, Wang G. Beneficial effects of growth hormone on bacterial translocation during the course of acute necrotizing pancreatitis in rats. Pancreas 2001;23:148156.

39. Shinzeki M, Takeyama Y, Ueda T, Yasuda T, Kishi S, Kuroda Y. Intraperitoneal administration of oxygenated perfluorochemical inhibits bacterial translocation associated with severe acute pancreatitis. Kobe J Med Sci 2003;49:17-24.

40. Czabotar PE, Lessene G, Strasser A, Adams JM. Control of apoptosis by the BCL-2 protein family: implications for physiology and therapy. Nat Rev Mol Cell Biol 2014;15:4963.

41. Pan H, Hong F, Radaeva S, Gao B. Hydrodynamic gene delivery of interleukin-22 protects the mouse liver from concanavalin A-, carbon tetrachloride-, and Fas ligand-induced injury via activation of STAT3. Cell Mol Immunol 2004;1:4349.

42. Feng D, Park O, Radaeva S, et al. Interleukin-22 ameliorates cerulein-induced pancreatitis in mice by inhibiting the autophagic pathway. Int J Biol Sci 2012;8:249-257.

43. Cox JH, Kljavin NM, Ota N, et al. Opposing consequences of IL-23 signaling mediated by innate and adaptive cells in chemically induced colitis in mice. Mucosal Immunol 2012;5:99-109.

44. Ki SH, Park O, Zheng M, et al. Interleukin-22 treatment ameliorates alcoholic liver injury in a murine model of chronic-binge ethanol feeding: role of signal transducer and activator of transcription 3. Hepatology 2010;52:1291-1300.

45. Chestovich PJ, Uchida Y, Chang W, et al. Interleukin-22: im- 
plications for liver ischemia-reperfusion injury. Transplantation 2012;93:485-492.

46. Nagalakshmi ML, Rascle A, Zurawski S, Menon S, de Waal Malefyt R. Interleukin-22 activates STAT3 and induces IL-10 by colon epithelial cells. Int Immunopharmacol 2004;4:679-691.

47. Gao B. Hepatoprotective and anti-inflammatory cytokines in alcoholic liver disease. J Gastroenterol Hepatol 2012;27 Suppl 2(Suppl 2):89-93. 